\title{
Relativistic Effects Appearing at Non-Relativistic Speeds
}

\author{
Kenji Kawashima* \\ Kanto Gakuin University, 1-50-1 Mutsuurahigashi, Kanazawa-ku, Yokohama, \\ Kanagawa 236-8501, Japan
}

\begin{abstract}
We study the center of energy (CE) before and after the separation of superposed wave from a moving medium (MM). It is assumed that two out-of-phase mechanical transverse waves propagating from the opposite directions on a medium moving at non-relativistic speeds are superposed and the superposed portion (SP) is separated from the MM at that moment. We consider the CE of the SP before and after the separation from the MM. The location of CE (LCE) of the SP seems to be at the center of it at the moment of superposition. The SP rotates due to the separation from the MM since the velocity of each portion symmetric with respect to the center of the SP is equal in magnitude and opposite in direction. The magnitudes of velocities of the symmetric portions become different as soon as the SP begins to rotate with the separation from the MM. Then the energies of their symmetric portions are not the same, so the LCE of the SP is not at the center of it. As a result, the LCE of it looks different before and after the separation from the MM. We must find a solution to keep the LCE of the SP constant. We propose that two out-of-phase mechanical transverse waves (MTWs) propagating from the opposite directions on a $\mathrm{MM}$ originally have hidden difference in relativistic energy and it suddenly appears within the range observable in Newtonian mechanics when the SP starts rotating. This means that the point of view of hidden difference in relativistic energy is necessary to keep the LCE constant.
\end{abstract}

Key words: hidden difference in relativistic energy, center of energy, time dilation, mechanical transverse wave, superposition of waves 


\section{Introduction}

It is regarded as one of the most fundamental principles of physics that the location of center of mass in an isolated frame of reference is always constant. From this principle, Einstein has derived the equivalence of mass and energy in special relativity (SR). ${ }^{1,2}$ According to it, the LCE is always constant in an isolated frame of reference. ${ }^{3-5}$ This is called the $\mathrm{CE}$ theorem in $\mathrm{SR} .^{3-6}$ The concept of $\mathrm{CE}$ is the relativistic generalization of center of mass because it includes not only rest energy but also all forms of energy. ${ }^{3}$ If the $\mathrm{CE}$ in a frame of reference is at rest, then its total momentum is zero. ${ }^{3,6,7}$ Here the CE theorem is related to the law of conservation of momentum. Furthermore, physical quantity like hidden momentum mainly in the electromagnetic field has been proposed from this theorem. ${ }^{7-12}$ The hidden momentum is a notion devised to prevent the change in energy density, in other words, to keep the LCE constant. ${ }^{8,13}$ In contrast, the concept like hidden energy has not been proposed.

We study hidden difference in relativistic energy that will be necessary to keep the LCE constant. This is irrelevant to the above hidden momentum in the electromagnetic field. An example that requires the concept of hidden difference in relativistic energy is the problem with the CE of the SP before and after the separation from the MM. We consider the superposition of two out-of-phase MTWs propagating from the opposite directions on a medium moving at non-relativistic speeds and the separation of the SP from the medium. The LCE of the SP seems to be at the center of it at the moment of superposition. The SP starts rotating with the separation from the MM because the velocity of each portion symmetric with respect to the center of it is equal in magnitude and opposite in direction. The magnitudes of velocities of the symmetric portions become different as soon as the SP begins to rotate at the same time as the separation from the MM. Hence, the energies of the symmetric portions are not same. As a result, the LCE of the SP is not at the center of it. This means that the LCE of the SP looks different before and after the separation from the MM. We can not find any previous work examining this. We must discover a solution to keep the LCE of the SP constant under non-relativistic speeds at which the medium moves.

To keep the LCE constant, we need to postulate that the two out-of-phase MTWs propagating from the opposite directions on the MM originally have the hidden difference in relativistic energy. It suddenly appears as the observable difference in energy even under non-relativistic speeds when the SP begins to rotate. Therefore, we apply SR to physical phenomenon under non-relativistic speeds to analyze this hidden difference in relativistic energy. 


\section{The LCE of Out-of-phase MTWs on a MM}

First of all, we assume two inertial frames of reference, $S^{\prime}$ and $S$, that are in a state of uniform relative motion. $S^{\prime}$ moves with constant non-relativistic velocity $V$ in the positive direction of the $x$ axis in $S$. Here the $x$ axis in $S$ and the $x^{\prime}$ axis in $S^{\prime}$ are on the same straight line. A box is stationary in $S^{\prime}$. Moreover, the box is equipped with the string overlapping with the $x^{\prime}$ axis in $S^{\prime}$.

We simultaneously move both ends of the string quickly up and down, in other words, along the $y^{\prime}$ axis perpendicular to the $x^{\prime}$ axis in $S^{\prime}$. We thereby generate two MTWs that have $1 / 2$ wavelength respectively and are symmetric with respect to the $x^{\prime}$ axis and $y^{\prime}$ axis in $S^{\prime}$. The two out-of-phase MTWs propagating in the opposite and advancing direction of the MM are generated above and below the medium that is in equilibrium, respectively. The magnitude of velocity of each portion symmetric with respect to the $x^{\prime}$ and $y^{\prime}$ axes is identical, so the symmetric portions have the same energy. Thus, the LCE of the two MTWs is constant and is on the $x^{\prime}$ axis overlapping with the medium in equilibrium (ME).

Thereafter, the two MTWs propagating from the opposite directions is superposed on the $x^{\prime}$ axis instantaneously and all of their energies become kinetic energies. At that moment, the magnitude of the transverse velocity (TV) of each portion symmetric with respect to the center of the SP is identical in $S^{\prime}$. Hence, those portions have the same kinetic energy. As a result, the LCE of the SP is on the $x^{\prime}$ axis in $S^{\prime}$, so it remains constant.

This, within the range observable, also seems to hold true for $S$ and hence the LCE of the SP looks located on the $x$ axis.

\section{The LCE of The SP Separated from the MM}

Here we separate the SP from the MM instantaneously. As mentioned above, when the two waves are superposed, the velocity of each portion symmetric with respect to the center of the SP is equal in magnitude and opposite in direction. Hence, the SP starts rotating clockwise due to the separation from the MM because its left and right sides have upward and downward velocities respectively. Then the SP becomes vertical with respect to the $x^{\prime}$ and $x$ axes momentarily. In this state, the distribution of the rest mass of the $\mathrm{SP}$ is symmetric with respect to each axis. For simplicity, we consider the SP in this state. 
The velocities of two portions above and below the $x^{\prime}$ axis are $v_{x}{ }^{\prime}$ and $-v_{x}{ }^{\prime}$ in $S^{\prime}$ respectively since the SP rotates clockwise. Supposed that each portion with the same mass is symmetric with respect to the $x^{\prime}$ axis. Hence, the magnitude of velocity of them is identical in $S^{\prime}$. Then, in $S$, the portion with $v_{x}{ }^{\prime}$ and that with $-v_{x}{ }^{\prime}$ are observed to have $V+v_{x}{ }^{\prime}$ and $V-v_{x}{ }^{\prime}$ respectively, so the former magnitude of velocity is large compared with the latter one of velocity. Therefore, in $S$, the energy of the portion above the $x$ axis is larger than that of the portion below the $x$ axis. In sum, the distribution of energy of the SP is not symmetric with respect to the $x$ axis in $S$. Let $E_{1}$ and $E_{2}$ be the energies at $y_{1}$ and $y_{2}$ symmetric with respect to the $x$ axis, respectively. Suppose that $y_{1}$ and $y_{2}$ are located above and below the $x$ axis, respectively. Moreover, let $y_{c}$ be the center of $y_{1}$ and $y_{2}$. It is on the $x$ axis. Then, since $E_{1}$ at $y_{1}$ is larger than $E_{2}$ at $y_{2}$, we obtain

$$
E_{1}\left(y_{1}-y_{c}\right)>E_{2}\left(y_{c}-y_{2}\right)
$$

This can be applied to any portion symmetric with respect to the $x$ axis. Let $E_{i}$ and $E_{j}$ be the energies at arbitrary $y$ coordinates $y_{i}$ and $y_{j}$ symmetric with respect to the $x$ axis, respectively. Moreover, suppose that $y_{i}$ and $y_{j}$ are located above and below the $x$ axis respectively. Then, since $E_{i}$ at $y_{i}$ is larger than $E_{j}$ at $y_{j}$, we have

$$
E_{i}\left(y_{i}-y_{c}\right)>E_{j}\left(y_{c}-y_{j}\right)
$$

where $y_{c}$ is the center of $y_{i}$ and $y_{j}$. Again, $y_{c}$ is on the $x$ axis.

From these facts, the LCE of the SP is not on the $x$ axis overlapping with the ME and is located above it. Consequently, in $S$, the LCE of the SP seems to be different before and after the separation from the MM.

By contrast, the production of the two out-of-phase MTWs propagating from the opposite directions on the medium results in the counterclockwise rotation of the box itself or other bodies. Suppose that one portion of the rotating body is above $x^{\prime}$ axis and the other portion of it is below the $x^{\prime}$ axis, respectively. The velocities of portions above and below the $x^{\prime}$ axis are $-v_{x}{ }^{\prime}$ and $v_{x}{ }^{\prime}$ in $S^{\prime}$ respectively since the body rotates counterclockwise. Again, supposed that each portion with the same mass is symmetric with respect to the $x^{\prime}$ axis. Hence, the magnitude of velocity of them is identical in $S^{\prime}$. Then, in $S$, the portion with $-v_{x}{ }^{\prime}$ and that with $v_{x}{ }^{\prime}$ are observed to have $V-v_{x}{ }^{\prime}$ and $V+v_{x}{ }^{\prime}$, respectively. Therefore, in $S$, the energy of a portion above the $x$ axis is small compared to that of a portion below the $x$ axis. This can also be applied to any portion 
symmetric with respect to the $x$ axis. From these, the LCE of the rotating body shifts below the $x$ axis.

In sum, the two shifts in the CE from the $x$ axis cancel each other. Hence, the LCE in $S$ is always on the $x$ axis, so it is constant. Nevertheless, the problem is that, within the range observable, the LCE of the medium does not seem to shift with the generation of the waves and thereafter suddenly seems to shift due to the separation of the SP from the MM.

\section{Physical Quantities Contributing to Relativistic Energy of the Two Waves}

We must find a solution to keep the LCE constant. To do so, we consider whether two out-of-phase MTWs propagating from the opposite directions on a MM originally have hidden difference in relativistic energy.

We start by analyzing the relativistic kinetic energies of the two out-of-phase waves propagating from the opposite directions on a MM. Two physical quantities that contribute to them are velocity and rest mass (RM) of each portion which is in wave motion (WM).

\subsection{Velocity contributing to relativistic kinetic energy (RKE)}

We analyze the velocity $v$ of a portion which is in WM in $S$. Let $v^{\prime}$ be the velocity of the corresponding portion observed from $S^{\prime}$. The velocity $v$ is one obtained by converting $v^{\prime}$ according to the Lorentz transformation. Again, suppose that $V$ is the velocity of $S^{\prime}$ in the positive direction of the $x$ axis in $S$.

On the basis of the law of velocity addition in SR, two components of $v$ of the portion, i.e., $v_{x}$ and $v_{y}$ are written as

$$
v_{x}=\frac{V+v_{x}^{\prime}}{1+V v_{x}^{\prime} / c^{2}}, \quad v_{y}=\frac{v_{y}^{\prime} \sqrt{1-V^{2} / c^{2}}}{1+V v_{x}^{\prime} / c^{2}}
$$

Moreover, since $v=\sqrt{\left(v_{x}^{2}+v_{y}^{2}\right)}$, substituting Eq. (3) into it, we have a transformation formula of velocity: 


$$
\begin{aligned}
v & =\sqrt{\left(\frac{V+v_{x}^{\prime}}{1+V v_{x}^{\prime} / c^{2}}\right)^{2}+\left(\frac{v_{y}^{\prime} \sqrt{1-V^{2} / c^{2}}}{1+V v_{x}^{\prime} / c^{2}}\right)^{2}} \\
& =\frac{\sqrt{V^{2}+2 V v_{x}^{\prime}+v_{x}^{\prime 2}+v_{y}^{\prime 2}\left(1-V^{2} / c^{2}\right)}}{1+V v_{x}^{\prime} / c^{2}} \\
& =\frac{\sqrt{V^{2}+2 V v_{x}{ }^{\prime}+v_{x}^{\prime 2}+v_{y}^{\prime 2}-V^{2} v_{y}^{\prime 2} / c^{2}}}{1+V v_{x}^{\prime} / c^{2}}
\end{aligned}
$$

In the case of MTWs, $v_{x}{ }^{\prime}=0$. Hence, the longitudinal velocity of each portion in WM observed from $S$ remains $V$. Substituting $v_{x}{ }^{\prime}=0$ for Eq. (4), we obtain

$$
v=\sqrt{V^{2}+v_{y}^{\prime 2}-V^{2} v_{y}^{\prime 2} / c^{2}} .
$$

As indicated above, the velocity of each portion of the wave contributing to the RKE obeys the Lorentz transformation as well as that of a particle.

\subsection{A relativistic peculiarity in the generation of MTWs}

Another physical quantity contributing to the RKE of the wave is the RM in WM. We need to indicate a relativistic peculiarity in the generation of MTWs before considering the RM in WM.

The continuous supply of energy from a wave source (WS) is done on a medium adjacent to it. In contrast, transfer of energy on the medium is performed at distant places from the WS. We assume the medium moving with constant velocity. According to the relativity of simultaneity, the time of supply of energy at the WS generally differs from the time of transfer of energy on the medium.

\subsection{RM contributing to $\mathrm{RKE}$}

The RM in WM is generally the amount obtained by multiplying unit length by mass density. Viewed from a different aspect, the RM in WM corresponds to the coordinate interval (CI) of the wave rather than the length of it.

Firstly, we consider the amount of RM of the wave propagating in the opposite direction of the MM in $S$. Let $W_{o}$ be its wave. Here we need to take the relativity of simultaneity in SR into account. Suppose that clocks are fixed at certain equal intervals 
along the $x$ and $x^{\prime}$ axes respectively. Moreover, we assume that they are synchronized in some way on each axis.

According to the relativity of simultaneity, the clocks at the back of the MM always go by fast compared to those at the front of it. This means that, in $S$, an event A in the back of the MM occurs earlier than one B in the front of it even if both of them take place in $S^{\prime}$ simultaneously. When a wave propagates in the opposite direction of the MM, the $x^{\prime}$ coordinate corresponding to the leading end of the wave (LEW) is at the back of the MM compared to that corresponding to the WS. Hence, the time on the former $x^{\prime}$ coordinate goes by faster than that on the latter $x^{\prime}$ coordinate. Let $x_{0}{ }^{\prime}$ and $-x_{1}{ }^{\prime}$ be the positions of the WS and LEW at a certain time $t_{1}{ }^{\prime}$ in $S^{\prime}$, respectively. When observing the wave in $S$, if the position $x_{0}$ and time $t_{1}$ of the WS coincide with $x_{0}{ }^{\prime}$ and $t_{1}{ }^{\prime}$ in $S^{\prime}$ respectively, then the LEW is not at $-x_{1}{ }^{\prime}$. The time on $-x_{1}{ }^{\prime}$ is not $t_{1}{ }^{\prime}$ and $t_{1}{ }^{\prime}$ is already past. Therefore, the LEW observed from $S$ has already passed through $-x_{1}{ }^{\prime}$ and propagates backward on the MM compared to that observed in $S^{\prime}$. When observing the LEW from $S$, it is at $-x_{2}{ }^{\prime}$ that is positioned farther from $x_{0}{ }^{\prime}$ compared with $-x_{1}{ }^{\prime}$. Also, at that moment, the clock on $-x_{2}{ }^{\prime}$ observed in $S$ shows $t_{2}{ }^{\prime}$. Here we can represent the CI by the absolute value. Then the coordinate interval of the portion in WM (CIPWM) observed from $S$ is $\left|-x_{2}-x_{0}\right|$, while that in $S^{\prime}$ is $\left|-x_{1}{ }^{\prime}-x_{0}{ }^{\prime}\right|$. Therefore, we obtain

$$
\left|-x_{2}-x_{0}\right|>\left|-x_{1}^{\prime}-x_{0}^{\prime}\right|
$$

Moreover, we assume that the portion corresponding to $\left|-x_{1}{ }^{\prime}-x_{0}{ }^{\prime}\right|$ has a TV $v_{y}{ }^{\prime}$ in $S^{\prime}$. Converting $v_{y}{ }^{\prime}$ in $S^{\prime}$ into the corresponding TV $v_{y}$ in $S$, from Eq. (3), $v_{y}=$ $v_{y}{ }^{\prime} \sqrt{1-V^{2} / c^{2}}$ since $v_{x}{ }^{\prime}=0$. As indicated above, the LEW in $S$ arrives at $-x_{2}$ when the TV of the portion becomes $v_{y}{ }^{\prime}$ in $S^{\prime}$. Hence, the CIPWM with $v_{y}^{\prime} \sqrt{1-V^{2} / c^{2}}$ is $\left|-x_{2}-x_{0}\right|$. As the result, we can find that the CIPWM having $v_{y}$ in $S, I_{o}$, is larger than one having $v_{y}{ }^{\prime}$ in $S^{\prime}, I^{\prime}$, i.e.,

$$
I_{o}>I^{\prime}
$$

Here the term, interval, does not denote the distance between the coordinates of the portion in WM, in other words, the length of the wave. On the other hand, a RM corresponds to the CI. Therefore, we can calculate the former using the latter. The RM of 
the portion having $v_{y}$ in $S, m_{o}$, is defined as

$$
m_{o}=I_{o} \rho^{\prime}
$$

where $\rho^{\prime}$ is RM per unit CI in $S^{\prime}$. Here $m_{o}$ in $S$ is determined using $\rho^{\prime}$ in $S^{\prime}$ because the RM corresponding to the CI is depended on the proper $\rho^{\prime}$ in $S^{\prime}$ relative to which the $\mathrm{ME}$ is at rest. The $\mathrm{RM} m^{\prime}$ having $v_{y}{ }^{\prime}$ in $S^{\prime}$ is equal to a value obtained by multiplying $I^{\prime}$ by $\rho^{\prime}$, i.e., $m^{\prime}=I^{\prime} \rho^{\prime}$. Then, since $I_{o}>I^{\prime}$ from inequality (7), we find that the RM $m_{o}$ having $v_{y}$ in $S$ is larger than that $m^{\prime}$ having $v_{y}{ }^{\prime}$ in $S^{\prime}$. The difference in the RM between the former and the latter results from that of the CI because $\rho^{\prime}$ is the same value in each frame of reference. Since its difference is proportional to the ratio of the $\mathrm{CI}$, we get

$$
m_{o}=m^{\prime} \frac{I_{o}}{I^{\prime}}>m^{\prime}
$$

The difference in the CI, in other words, that in the RM between $S$ and $S^{\prime}$, as indicated above, depends upon the time difference at the coordinates on the $x^{\prime}$ axis observed from $S$. This time difference is determined according to the velocity $V$ of $S^{\prime}$ relative to $S$.

Secondly, we analyze the amount of RM of the wave propagating in the advancing direction of the MM in $S$. Let $W_{a}$ be its wave. In contrast to $W_{o}$, in $W_{a}$, the $x^{\prime}$ coordinate corresponding to the LEW is at the front of the MM compared with that corresponding to the WS. Hence, the time on the former $x^{\prime}$ coordinate goes by slower than that on the latter $x^{\prime}$ coordinate. Then, if the position and time of the WS in $S$ coincide with them of the WS in $S^{\prime}$ respectively, then the LEW in $S^{\prime}$ propagates forward on the MM compared to that observed from $S$. In other words, the latter is at the back of the MM compared with the former. This means that the CIPWM in $S$ is smaller than that in $S^{\prime}$. Let $-v_{y}{ }^{\prime}$ and $-v_{y}$ be the transverse velocities of the portions corresponding to each CIPWM in $S^{\prime}$ and $S$, respectively. The latter velocity is also one obtained by converting $-v_{y}{ }^{\prime}$ according to the Lorentz transformation. Then the CIPWM having $-v_{y}$ in $S, I_{a}$, is smaller than that having $-v_{y}{ }^{\prime}$ in $S^{\prime}, I^{\prime}$, i.e.,

$$
I_{a}<I^{\prime}
$$

The RM of the portion having $-v_{y}$ in $S, m_{a}$, is defined as 


$$
m_{a}=I_{a} \rho^{\prime}
$$

Furthermore, since $I_{a}<I^{\prime}$ from inequality (10), we find that the RM $m_{a}$ having $-v_{y}$ in $S$ is smaller than that $m^{\prime}$ having $-v_{y}{ }^{\prime}$ in $S^{\prime}$. Again, since its difference is proportional to the ratio of the CI, we obtain

$$
m_{a}=m^{\prime} \frac{I_{a}}{I^{\prime}}<m^{\prime}
$$

We assume that the magnitudes of velocities of $v_{y}{ }^{\prime}$ and $-v_{y}{ }^{\prime}$ are the same. Thus, those of velocities of $v_{y}$ and $-v_{y}$ are also the same. Then combining expressions (7) and (10) yields

$$
I_{o}>I^{\prime}>I_{a}
$$

Furthermore, combining expressions (9) and (12), we obtain

$$
m_{o}>m^{\prime}>m_{a}
$$

Consequently, the RM of a portion of $W_{o}$ is larger than that of a portion of $W_{a}$ when each portion have the TV of the same magnitude.

Inequality (13) and (14) hold true for any CIPWM having the TV of the same magnitude. Therefore, we firstly get

$$
I_{T o}>I_{T}^{\prime}>I_{T a}
$$

where $I_{T o}$ and $I_{T a}$ are the total CIPWM of $W_{o}$ and $W_{a}$ in $S$ respectively and $I_{T}{ }^{\prime}$ is that of one wave in $S^{\prime}$. Secondly, we obtain

$$
M_{T o}>M_{T}^{\prime}>M_{T a}
$$

where $M_{T o}$ and $M_{T a}$ are the total RM of $W_{o}$ and $W_{a}$ in $S$ respectively and $M_{T}{ }^{\prime}$ is that of one wave in $S^{\prime}$.

Here we need to bear in mind that we consider and compare only the amount of RM that is in WM. Total RM including the portion that are not in WM, needless to say, is 
invariant for the Lorentz transformation.

\section{Hidden Difference in Relativistic Energy of the Two Waves}

\subsection{The amount of RKE}

We firstly compare the RKE of a portion of $W_{o}$ with that of a portion of $W_{a}$. The RKE $E$ of a portion with RM $m$ and velocity $v$ is generally given by

$$
E=m c^{2}\left(\frac{1}{\sqrt{1-v^{2} / c^{2}}}-1\right)
$$

where $c$ is the speed of light. Substituting Eq. (5) into Eq. (17), we have

$$
E=m c^{2}\left\{\frac{1}{\sqrt{1-\left(\sqrt{V^{2}+v_{y}^{\prime 2}-V^{2} v_{y}^{\prime 2} / c^{2}}\right)^{2} / c^{2}}}-1\right\} .
$$

Here let $m_{o 1}$ and $m_{a 1}$ be the RM of a portion of $W_{o}$ and that of a portion of $W_{a}$, respectively. Moreover, let $v_{y}$ be the TV of the portion with $m_{o 1}$. Similarly, let $-v_{y}$ be the TV of the portion with $m_{a 1}$. These $v_{y}$ and $-v_{y}$ are the velocities obtained by converting $v_{y}{ }^{\prime}$ and $-v_{y}{ }^{\prime}$ observed from $S^{\prime}$ according to the Lorentz transformation respectively. Again, from Eq. (3), $v_{y}=v_{y}^{\prime} \sqrt{1-V^{2} / c^{2}}$ and $-v_{y}=-v_{y}^{\prime} \sqrt{1-V^{2} / c^{2}}$ since $v_{x}{ }^{\prime}=0$. Supposed that the portions with $m_{o 1}$ and $m_{a 1}$ have the same displacement in the direction of $y$ axis. Hence, each magnitude of TV is also identical. Again, each longitudinal velocity is the same $V$. Then the RKE $E_{K m o 1}$ of the portion having $m_{o 1}$ and that $E_{K m a 1}$ of the portion having $m_{a 1}$ are given by the following equations:

$$
E_{K m o 1}=m_{o 1} c^{2}\left\{\frac{1}{\sqrt{1-\left(\sqrt{V^{2}+v_{y}^{\prime 2}-V^{2} v_{y}^{\prime 2} / c^{2}}\right)^{2} / c^{2}}}-1\right\}
$$


and

$$
E_{K m a 1}=m_{a 1} c^{2}\left\{\frac{1}{\sqrt{1-\left(\sqrt{V^{2}+\left(-v_{y}^{\prime}\right)^{2}-V^{2}\left(-v_{y^{\prime}}\right)^{2} / c^{2}}\right)^{2} / c^{2}}}-1\right\}
$$

From inequalities (14), $m_{o 1}>m_{a 1}$. In contrast, other physical quantities contributing to the RKE are the same. Needless to say, in Eqs. (19) and (20), even if the signs of $v_{y}$ and $-v_{y}$ in two equations are different, their squared values are the same. Therefore, we find

$$
E_{K m o 1}>E_{K m a 1}
$$

Let $E_{T K o}$ and $E_{T K a}$ be the total RKE of $W_{o}$ and that of $W_{a}$, respectively. $E_{T K o}$ is given by

$$
E_{T K o}=E_{K m o 1}+E_{K m o 2}+E_{K m o 3}+\cdots=\sum_{i=1}^{n} E_{K m o i} .
$$

Likewise, $E_{T K a}$ is expressed as

$$
E_{T K a}=E_{K m a 1}+E_{K m a 2}+E_{K m a 3}+\cdots=\sum_{i=1}^{n} E_{K m a i} .
$$

Inequality (21) holds true for any portion having the velocity of the same magnitude (VSM). Therefore, the RKE of each term in Eq. (22) is larger than that of the corresponding term in Eq. (23) on the premise that each portion of the corresponding terms has the VSM. Consequently, we find the following inequality

$$
E_{T K o}>E_{T K a}
$$

This difference due to that of the RM in WM does not appear within the range observable during WM. Therefore, $E_{T K o}-E_{T K a}$ is the hidden difference in relativistic energy. 
Here we consider the above difference in relativistic energy from the point of view of the Lorentz transformation with respect to energy and momentum. A general relational expression of energy and momentum is given by

$$
E^{2}=m^{2} c^{4}+c^{2} p^{2}
$$

where $m$ and $p$ are the RM and momentum of a portion respectively and $c$ denotes the speed of light. Let $E_{o 1}, p_{o 1}$ and $m_{o 1}$ be the energy, momentum and RM of a portion of $W_{o}$, respectively. Similarly, let be $E_{a 1}, p_{a 1}$ and $m_{a 1}$ the energy, momentum and RM of a portion of $W_{a}$, respectively. Suppose that the two portions have the VSM. From inequalities (14), $m_{o 1}>m_{a 1}$. Furthermore, $p_{o 1}^{2}>p_{a 1}^{2}$ since $m_{o 1}>m_{a 1}$. Consequently, we get

$$
E_{o 1}^{2}=m_{o 1}^{2} c^{4}+c^{2}{p_{o 1}}^{2}>E_{a 1}^{2}=m_{a 1}^{2} c^{4}+c^{2} p_{a 1}^{2}
$$

Hence, the RM and momentum of any portion of $W_{o}$ are larger than those of any portion of $W_{a}$ if each portion has the VSM. Here let $E_{t o}$ and $E_{t a}$ be the total energy of the portions of $W_{o}$ and that of the portions of $W_{a}$, respectively. $E_{\text {to }}$ is given by

$$
\begin{aligned}
E_{t o}{ }^{2} & =\left(m_{o 1}{ }^{2} c^{4}+c^{2}{p_{o 1}}^{2}\right)+\left({m_{o 2}}^{2} c^{4}+c^{2}{p_{o 2}}^{2}\right)+\left({m_{o 3}}^{2} c^{4}+c^{2}{p_{o 3}}^{2}\right)+\cdots \\
& =\sum_{i=1}^{n}\left(m_{o i}{ }^{2} c^{4}+c^{2} p_{o i}^{2}\right)
\end{aligned}
$$

Likewise, $E_{t a}$ is expressed as

$$
\begin{aligned}
E_{t a}{ }^{2} & =\left(m_{a 1}{ }^{2} c^{4}+c^{2}{p_{a 1}}^{2}\right)+\left(m_{a 2}{ }^{2} c^{4}+c^{2}{p_{a 2}}^{2}\right)+\left(m_{a 3}{ }^{2} c^{4}+c^{2} p_{a 3}{ }^{2}\right)+\cdots \\
& =\sum_{i=1}^{n}\left(m_{a i}{ }^{2} c^{4}+c^{2} p_{a i}^{2}\right)
\end{aligned}
$$

The RKE of each term in Eq. (27) is larger than that of the corresponding term in Eq. (28) on the premise that each portion of the corresponding terms has the VSM. After all, similarly to inequality (24), the relationship between $E_{t o}{ }^{2}$ and $E_{t a}{ }^{2}$ is as follows: 


$$
E_{t o}^{2}>E_{t a}^{2}
$$

\subsection{The amount of potential energy (PE)}

Secondly, we analyze the PE of the two waves. A portion in WM with a certain displacement in the direction of the $y^{\prime}$ axis in $S^{\prime}$ has not only the corresponding TV but also a constant proper PE. This PE is stored in the portion corresponding to a CI. Thus, the amount of PE is depended on the CIPWM. This holds true even though the forms of each portion, in other words, their waveforms look different in $S$. When observing two CIPWMs with the VSM in $S$, as indicated above, each CIPWM included in $W_{o}$ and $W_{a}$ is different.

Again, let $I_{o}$ and $I_{a}$ be the CI of a portion included in $W_{o}$ and that of a portion included in $W_{a}$, respectively. From inequalities (13), $I_{o}>I_{a}$. Therefore, for the PE $E_{P I_{o}}$ stored in the portion corresponding to $I_{o}$ and that $E_{P I_{a}}$ stored in the portion corresponding to $I_{a}$, we obtain

$$
E_{P I_{o}}>E_{P I_{a}}
$$

Furthermore, inequality (30) is true for any $\mathrm{CI}$ having an arbitrary displacement and the corresponding TV. Let $I_{T o}$ and $I_{T a}$ be the total CIPWM including $I_{O}$ and that including $I_{a}$, respectively. From inequalities (15), $I_{T o}>I_{T a}$. Therefore, for the total PE $E_{T P o}$ corresponding to $I_{T o}$ and that $E_{T P a}$ corresponding to $I_{T a}$, we get

$$
E_{T P o}>E_{T P a}
$$

Inequality (31) tells us that the total PE of $W_{o}$ is larger than one of $W_{a}$. This difference due to that of the CI in WM does not appear within the range observable during WM. Therefore, $E_{T P O}-E_{T P a}$ is also the hidden difference in relativistic energy.

\section{Appearance of Hidden Difference in Relativistic Energy}

We compared the relativistic kinetic and potential energy, i.e., the total relativistic energy $E_{T o}$ of $W_{o}$ with that $E_{T a}$ of $W_{a}$. As already indicated, the former and latter waves correspond to those which have been generated above and below the $x$ axis overlapping with the ME, respectively. Combining inequalities (24) and (31) yields 


$$
E_{T o}=E_{T K o}+E_{T P o}>E_{T a}=E_{T K a}+E_{T P a} .
$$

These expressions are derived from the difference in the CI of the two waves and the corresponding difference in RM.

From Eq. (32), the CE of the two waves has been located above $x$ axis overlapping with the ME since before the separation of the SP from the MM although this has not been within the range observable under non-relativistic speeds. We conclude that the two waves have hidden difference in relativistic energy and its difference suddenly appears due to the separation of the SP from the MM. This means that the unobserved difference in energy, $E_{T o}-E_{T a}$, appears as that in observable kinetic energy. In other words, this shows that relativistic effect normally non-observable can be observed within Newtonian mechanics.

\section{Conclusion}

We studied a solution to keep the LCE constant in the phenomenon that the superposed wave separates from the MM. We proposed that the two out-of-phase MTWs propagating from the opposite directions on a medium moving at non-relativistic speeds have hidden difference in relativistic energy. Its difference suddenly appears within the range observable when the SP begins to rotate due to the separation from the MM. The LCE observed from $S$ remains constant because that of the two waves has originally been above $x$ axis overlapping with the ME. Consequently, the viewpoint of hidden difference in relativistic energy is necessary to keep the LCE constant.

We conducted a qualitative research on appearance of hidden difference in relativistic energy. Quantitative researches in this regard are expected in the future.

E-mail: kawaken@kanto-gakuin.ac.jp

\section{References}

${ }^{1}$ A. Einstein, "Ist die Trägheit eines Körpers von seinem Energieinhalt abhängig?, " Ann. Phys. 18, 639-641 (1905).

2 A. Einstein, "Das Prinzip von der Erhaltung der Schwerpunktsbewegung und die 
Trägheit der Energie," Ann. Phys. 20, 627-633 (1906).

${ }^{3}$ D. J. Griffiths, "Resource Letter EM-1: Electromagnetic Momentum, ” Am. J. Phys. 80 (1) , 7- 18 (2012).

${ }^{4}$ T. H. Boyer, "Interaction of a magnet and a point charge: Unrecognized internal electromagnetic momentum, ” Am. J. Phys. 83 (5), 433-442 (2015).

${ }^{5}$ S. Coleman and J. H. Van Vleck, "Origin of "Hidden Momentum Forces" on Magnets," Phys. Rev. 171 (5), 1370-1375 (1968).

${ }^{6}$ D. Babson, S. P. Reynolds, R. Bjokquist, and D. J. Griffiths, "Hidden momentum, field momentum, and electromagnetic impulse, ” Am. J. Phys. 77 (9), 826-833 (2009).

${ }^{7}$ W. H. Furry, "Examples of Momentum Distributions in the Electromagnetic Field and in Matter, ” Am. J. Phys. 37 (6), 621 - 636 (1969).

${ }^{8}$ W. Shockley and R. P. James, “ 'Try simplest cases' discovery of 'hidden momentum' forces on 'magnetic currents'," Phys. Rev. Lett. 18 (20), 876-879 (1967).

${ }^{9} \mathrm{~V}$. Hnizdo, "Hidden momentum and the electromagnetic mass of a charge and current carrying body, ” Am. J. Phys. 65 (1), 55-65 (1997).

${ }^{10}$ A. L. Kholmetskii, and T. Yarman, "The semi-classical limit of the Aharonov-Bohm effect: The actualized approach, ” Eur. Phys. J. Plus 128, 30-40 (2013).

${ }^{11}$ A. L. Kholmetskii, O. V. Missevitch, and T. Yarman, "Classical electrodynamics in material media and relativistic transformation of magnetic dipole moment, " Eur. Phys. J. Plus 131, 316-325 (2016) .

${ }^{12}$ G. Spavieri, "Role of the electromagnetic momentum in the spin-orbit interaction," Eur. Phys. J. D 70, 263- 270 (2016).

${ }^{13} \mathrm{~V}$. Hnizdo, "Conservation of linear and angular momentum and the interaction of a moving charge with a magnetic dipole, ” Am. J. Phys. 60 (3), 242-246 (1992). 